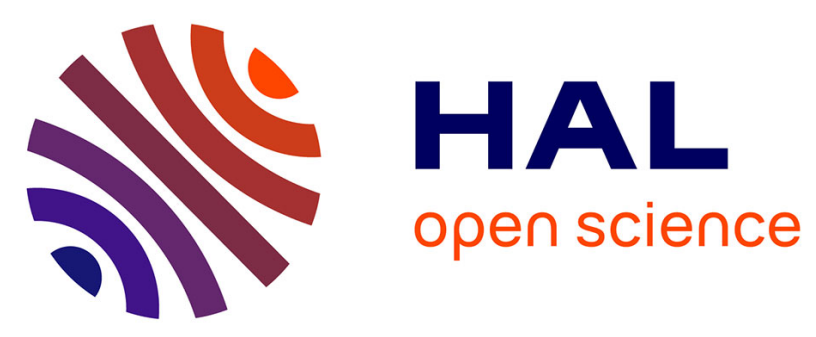

\title{
Temporal trends of population viral suppression in the context of Universal Test and Treat: the ANRS 12249 TasP trial in rural South Africa
}

Joseph Larmarange, Mamadou Diallo, Nuala Mcgrath, Collins Iwuji, Mélanie Plazy, Rodolphe Thiébaut, Frank Tanser, Till Bärrnighausen, Joanna

Orne-gliemann, Deenan K Pillay, et al.

\section{To cite this version:}

Joseph Larmarange, Mamadou Diallo, Nuala Mcgrath, Collins Iwuji, Mélanie Plazy, et al.. Temporal trends of population viral suppression in the context of Universal Test and Treat: the ANRS 12249 TasP trial in rural South Africa. Journal of the International AIDS Society, 2019, 22 (10), 10.1002/jia2.25402 . hal-03161847v3

\section{HAL Id: hal-03161847}

\section{https://hal-amu.archives-ouvertes.fr/hal-03161847v3}

Submitted on 18 May 2020

HAL is a multi-disciplinary open access archive for the deposit and dissemination of scientific research documents, whether they are published or not. The documents may come from teaching and research institutions in France or abroad, or from public or private research centers.
L'archive ouverte pluridisciplinaire HAL, est destinée au dépôt et à la diffusion de documents scientifiques de niveau recherche, publiés ou non, émanant des établissements d'enseignement et de recherche français ou étrangers, des laboratoires publics ou privés. 


\title{
RESEARCHARTICLE
}

\section{Temporal trends of population viral suppression in the context of Universal Test and Treat: the ANRS 12249 TasP trial in rural South Africa}

Joseph Larmarange 1,2,\$ (D), Mamadou H Diallo ${ }^{1}$, Nuala McGrath ${ }^{3,4,5}$ (D), Collins Iwuji $i^{2,5,6}$ (D), Mélanie Plazy ${ }^{7}$, Rodolphe Thiébaut ${ }^{7}$, Frank Tanser ${ }^{3}$, Till Bärnighausen ${ }^{2,8,9}$, Joanna Orne-Gliemann" ${ }^{7}$, Deenan Pillay 2,10**, François Dabis ${ }^{7 *}$ and for the ANRS 12249 TasP Study Group

§Corresponding author: Joseph Larmarange, Centre Population et Développement, Université Paris Descartes, 45 rue des Saints-Pères, 75006 Paris, France. Tel: +33 6620651 82. (joseph.larmarange@ceped.org)

*These authors are joint senior authors on this work.

"Membership of the ANRS 12249 TasP trial group is provided in the Acknowledgments.

\begin{abstract}
Introduction: The universal test-and-treat (UTT) strategy aims to maximize population viral suppression (PVS), that is, the proportion of all people living with HIV (PLHIV) on antiretroviral treatment (ART) and virally suppressed, with the goal of reducing HIV transmission at the population level. This article explores the extent to which temporal changes in PVS explain the observed lack of association between universal treatment and cumulative HIV incidence seen in the ANRS 12249 TasP trial conducted in rural South Africa.

Methods: The TasP cluster-randomized trial (2012 to 2016) implemented six-monthly repeat home-based HIV counselling and testing (RHBCT) and referral of PLHIV to local HIV clinics in $2 \times 11$ clusters opened sequentially. ART was initiated according to national guidelines in control clusters and regardless of CD4 count in intervention clusters. We measured residency status, HIV status, and HIV care status for each participant on a daily basis. PVS was computed per cluster among all resident PLHIV ( $\geq 16$, including those not in care) at cluster opening and daily thereafter. We used a mixed linear model to explore time patterns in PVS, adjusting for sociodemographic changes at the cluster level.

Results: 8563 PLHIV were followed. During the course of the trial, PVS increased significantly in both arms (23.5\% to $46.2 \%$ in intervention, $+22.8, p<0.001 ; 26.0 \%$ to $44.6 \%$ in control, $+18.6, p<0.001)$. That increase was similar in both arms $(p=$ 0.514). In the final adjusted model, PVS increase was most associated with increased RHBCT and the implementation of local trial clinics (measured by time since cluster opening). Contextual changes (measured by calendar time) also contributed slightly. The effect of universal ART (trial arm) was positive but limited.

Conclusions: PVS was improved significantly but similarly in both trial arms, explaining partly the null effect observed in terms of cumulative HIV incidence between arms. The PVS gains due to changes in ART-initiation guidelines alone are relatively small compared to gains obtained by strategies to maximize testing and linkage to care. The achievement of the 90-90-90 targets will not be met if the operational and implementational challenges limiting access to care and treatment, often context-specific, are not properly addressed. Clinical trial number: NCT01509508 (clinicalTrials.gov)/DOH-27-0512-3974 (South African National Clinical Trials Register).
\end{abstract}

Keywords: HIV; antiretroviral therapy; sustained viral suppression; retention in care; population health; South Africa

Additional information may be found under the Supporting Information tab for this article.

Received 7 March 2019; Accepted 3 September 2019

Copyright $\odot 2019$ The Authors. Journal of the International AIDS Society published by John Wiley \& Sons Ltd on behalf of the International AIDS Society.

This is an open access article under the terms of the Creative Commons Attribution License, which permits use, distribution and reproduction in any medium, provided the original work is properly cited.

\section{1 | INTRODUCTION}

Antiretroviral treatment (ART), when taken early has several benefits, both in terms of morbidity and mortality [1,2] and in terms of reduction in HIV sexual transmission [3].
Mathematical modelling work suggested that a universal testand-treat (UTT) strategy (i.e. HIV testing of all adult members of a community followed by immediate ART initiation of those tested positive) could reduce HIV incidence at the population level and ultimately eliminate HIV transmission in South Africa 
[4]. Observational data from rural KwaZulu-Natal, South Africa, demonstrated a strong inverse association between ART coverage and HIV incidence [5]. Implementing a UTT strategy involves removing eligibility criteria for ART initiation and improving all steps of the "cascade of HIV care" [6,7] to maximize the proportion of people living with HIV (PLHIV) on ART and virally suppressed, that is, to increase population viral suppression (PVS).

Several research projects, including randomized controlled trials in Southern and Eastern Africa, have evaluated field efficacy of UTT [8-10]. The ANRS 12249 TasP trial, conducted in rural South Africa, was the first to yield results on impacts of universal ART on new HIV infections at the population level [11]. The underlying hypothesis of the TasP trial was that the implementation of early ART, regardless of immunological or clinical staging, will improve PVS, leading to a reduction in HIV incidence at population-level. However, some TasP intervention components (particularly HIV testing and local trial clinics) were implemented in both arms and could also have had positive effects on PVS in the control arm, reducing differences between arms.

Here we investigate the following questions: did PVS improve longitudinally during the trial? Were there differences by trial arm in level and/or temporal trend? Were changes (if any) mainly associated with secular changes in contextual factors (independent of the trial) or due to the trial activities? Were the same effects observed at each step of the HIV care cascade?

\section{METHODS}

\section{1 | Study setting and design}

The TasP trial was a two-arm cluster-randomized trial implemented by the Africa Health Research Institute (AHRI) in Hlabisa sub-district, KwaZulu-Natal, South Africa, in a rural area with approximately 28,000 isiZulu-speaking resident adults. Adult HIV prevalence in the sub-district was approximately $30 \%$ at the time of study design $[12,13]$. Hlabisa sub-district is characterized by frequent migration [14,15], low marital rates and late marriage [16]. One-tenth of adults are employed [11]. The trial protocol and study procedures have previously been reported in detail $[8,17]$.

The trial was implemented from March 2012 to June 2016 using a phased approach: four clusters were opened in 2012, six additional clusters opened in 2013 and 12 in 2014; all 22 clusters $(2 \times 11)$ were followed until mid-2016. Each cluster was designed to correspond to approximately 1000 resident adults.

In both arms, HIV counsellors visited all households and enumerated all resident adult ( $\geq 16$ years) household members (initial census in first survey round). At each subsequent semiannual home-based survey round, all households were (re)visited and the resident adult household member list was updated. Exits (including deaths and outmigration from the trial area) were documented as reported by other household members.

Eligible individuals providing written informed consent in isiZulu responded to a sociodemographic and sexual behaviour questionnaire and gave a finger prick sample collected as a dried blood spot (DBS), used for HIV incidence estimation.
HIV counsellors also offered individuals point-of-care rapid HIV counselling and testing. All trial participants identified as HIV positive (through rapid HIV tests or self-reports) were referred to local trial clinics situated in the trial clusters in which they lived, located at less than 45 minutes walking distance. From May 2013, support for linkage to trial clinics through phone calls and home visits by dedicated trial teams was offered to individuals not linked to care within three months after referral.

In the control cluster trial clinics, HIV-positive adults were offered ART according to national guidelines (initially CD4 count $\leq 350$ cells $/ \mathrm{mm}^{3}$, then $\leq 500$ cells $/ \mathrm{mm}^{3}$ from January 2015). In the intervention-cluster trial clinics, all HIV-positive adults were offered opportunities to begin ART immediately regardless of CD4 count or clinical staging. The trial area was also served by three local governmental clinics providing HIV testing, HIV care and ART according to national guidelines only [18]. HIV-positive participants in both arms could opt to receive HIV care in local governmental clinics or transfer to trial clinics.

The Biomedical Research Ethics Committee (BREC), University of KwaZulu-Natal, South Africa (BFC 104/11) and the Medicines Control Council of South Africa approved the trial. The trial was also registered on ClinicalTrials.gov: NCT01509508 and South African National Clinical Trials Register: DOH-27-0512-3974.

\subsection{Data sources}

The main data source for this analysis was the trial database, which provided information on trial registrations and trial exits; uptake and results of home-based rapid HIV testing; third generation ELISA HIV serological results from DBS; and clinic visits, ART prescriptions and viral loads of PLHIV seen in trial clinics.

Two additional data sources captured information from PLHIV seen in local governmental clinics: (a) viral loads and CD4 counts from the National Health Laboratory Service (NHLS); and (b) ART clinic visits and ART prescriptions from the AHRI clinical database (ACCDB) managed by the Hlabisa Department of Health and AHRI. Both NHLS and the ACCDB database contain data from Hlabisa local governmental clinics since 2004 [18]. Matching between trial, NHLS and ACCDB databases used probabilistic scores based on first names, last names, dates of birth, South African ID numbers and cellphone numbers. Database matching was approved by the BREC in March 2013 (protocol amendment 4).

\section{3 $\quad$ Daily statuses}

To facilitate the alignment of data according to different timelines (calendar time, time since cluster opening at the cluster level, time spent within the trial or within HIV care at the individual level), we estimated residency, HIV status and HIV care status (if resident and HIV positive) each day for all trial-registered individuals. We did this by combining information across all the data sources for the study, and linearly interpolating values between dates when data were observed. For changes in discrete variables, we used random imputation methods (random point approach). For details of the data combination and imputation process, see Supplementary materials. 
Individuals with no observed HIV status (i.e. with no data on HIV status) were excluded from analyses.

HIV care statuses were defined as (i) undiagnosed; (ii) diagnosed but not actively in care (i.e. never in care or lost-to-follow-up from care); (iii) actively in care but not on ART; (iv) on ART but not virally suppressed (undocumented viral load or viral load over 400 copies $/ \mathrm{mL}$ ); and (v) in care, on ART and documented viral suppression.

\subsection{Outcome definitions}

As all clusters were not opened simultaneously, and have therefore different observation periods (Figure 1), PVS was computed daily per trial cluster, from the end of the initial population census (to ensure that the population cohort is complete) to the beginning of the last survey round (to ensure that each household was revisited at least once, with opportunities to document any exit from the population cohort). In addition, a PVS value at cluster opening was estimated based on the individuals' situation at the initial population census.

For a given cluster at a given date, we defined PVS as the proportion being in care, on ART and virally suppressed among all resident adult PLHIV in that cluster at that date.
To better understand PVS trends, PVS could be disaggregated in four sub-indicators corresponding to different steps of the HIV care cascade: (a) proportion of diagnosed among all resident PLHIV; (b) proportion in-care among those diagnosed; (c) proportion on ART among those in care; and (d) proportion virally suppressed among those on ART. Regarding the UNAIDS's 90-90-90 framework [19], the first 90 is (a), the second is $(b) \times(c)$, the third is $(d)$, and PVS is $(a) \times(b) \times(c)$ $\times(d)$.

\subsection{Statistical analysis}

For descriptive analysis, PVS was compared by arm and at five different time points (cluster opening, 1 January 2013, 2014, 2015 and 2016). Proportions were compared by arm and between dates. Difference-in-differences were also computed. This analysis was stratified by year of cluster opening. To assess the bivariate effect of the trial arm on end-line PVS, we performed an analysis of the covariance (ANCOVA) by modelling end-line PVS according to trial arm and controlling for PVS at cluster opening [20].

To explore cluster-level PVS trends, we used linear models with cluster-day data. In model 1, we considered calendar
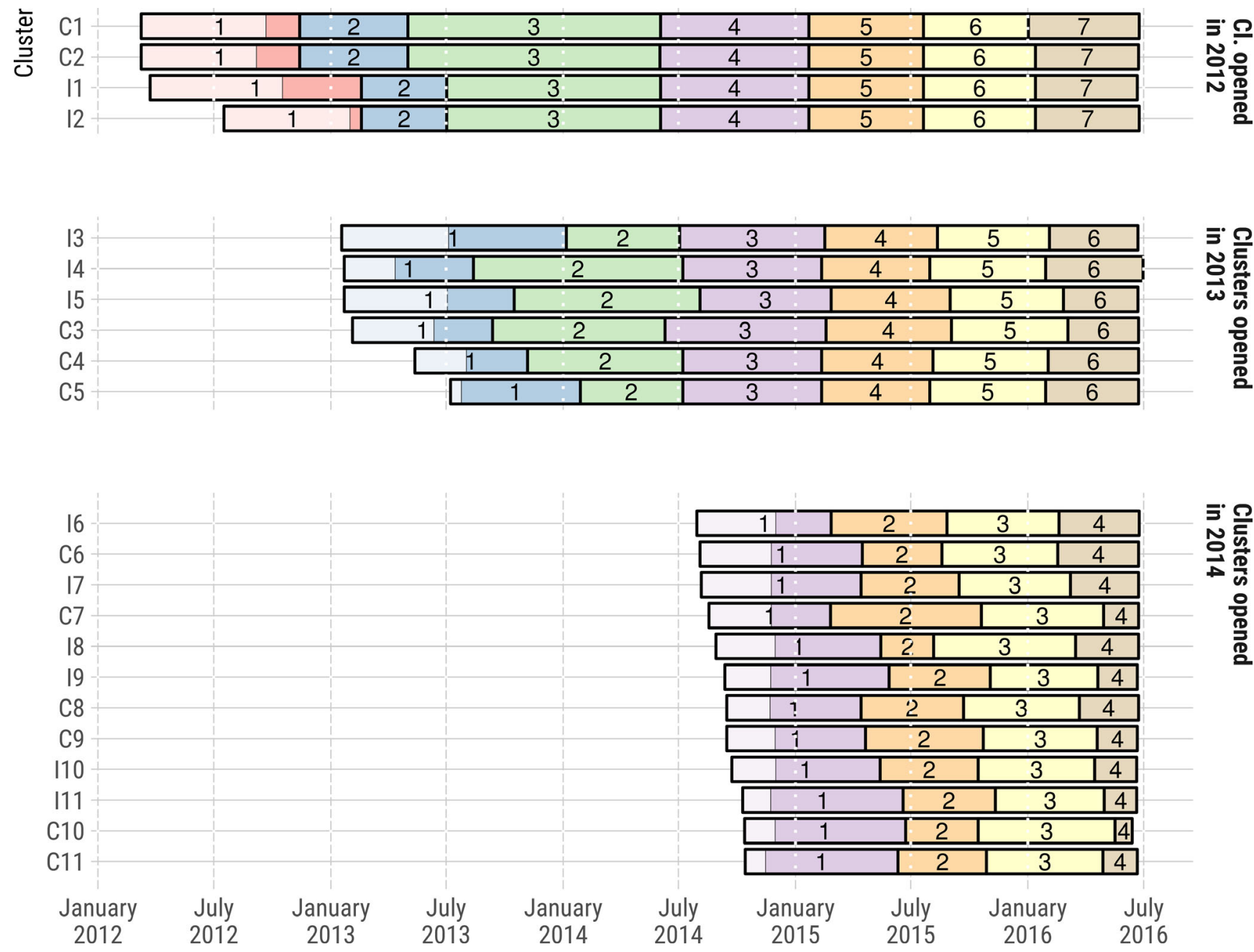

Figure 1. Dates of home-based survey rounds activities by clusters, ANRS 12249 TasP trial (2012 to 2016).

The light areas in round 1 indicate the time required to complete the initial census of the resident population. 
years (continuous variable), years since cluster opening (continuous variable), trial arm and interaction between trial arm and years since cluster opening. In model 2, we adjusted for cluster-level sociodemographic characteristics and HIV prevalence by introducing the following proportions computed for each cluster and at each given date among the resident PLHIV population: males; 16 to 29 years old; $\geq 60$ years old; single (never married and not engaged); employed; students; with at least second educational level; belonging to a household categorized as poor (see Supplementary materials); and observed HIV prevalence. All models were weighted by the number of resident PLHIV (time-dependent).

A similar approach was used to explore trends in different sub-components of the HIV care cascade.

All analyses were performed using $R$ version 3.4.4 [21]. To take into account the small numbers of clusters, we computed p-values and confidence intervals $(\mathrm{Cl})$ using the wild cluster bootstrapped t-statistics, as suggested by Cameron et al. [22] and implemented in clusterSEs package. This method was used both for descriptive analysis (proportions comparison and difference-in-differences) and for multivariate analysis (linear models).

\subsection{Sensitivity analysis}

To account for potential nonlinear trends, we also estimated models with three coefficients for calendar years (one each for 2012 to 2013, 2014, and 2015 to 2016) and three coefficients for years since cluster opening (first year, second, third/fourth).

We undertook sensitivity analyses to assess any potential bias of excluding individuals whose HIV status was not observed. A logistic regression was computed among individuals with an observed HIV status to model the probability of being HIV infected according to the cluster, sociodemographic characteristics, calendar time and time spent within the population cohort. This model was then used to predict the HIV status of individuals with no observed status. Those predicted to be HIV positive (probability $>50 \%$ ) were incorporated in the computation of PVS, considering that they were not virally suppressed (approach A). We also computed a multinomial logistic model to predict the care status of those imputed to be HIV positive (approach B).

\section{RESULTS}

During the trial 28,419 adults were registered over; 173 individuals exited the trial area before the initial census of their cluster ended or were registered during the last survey round. Among the remaining 28,246 individuals: HIV status was undocumented for 2612 (9\%), and 17,071 (60\%) remained HIV negative over the analysis period; thus, 8563 individuals were resident PLHIV population over the analysis period and included in the analysis (3940 in intervention arm and 4623 in control arm). Observed HIV prevalence did
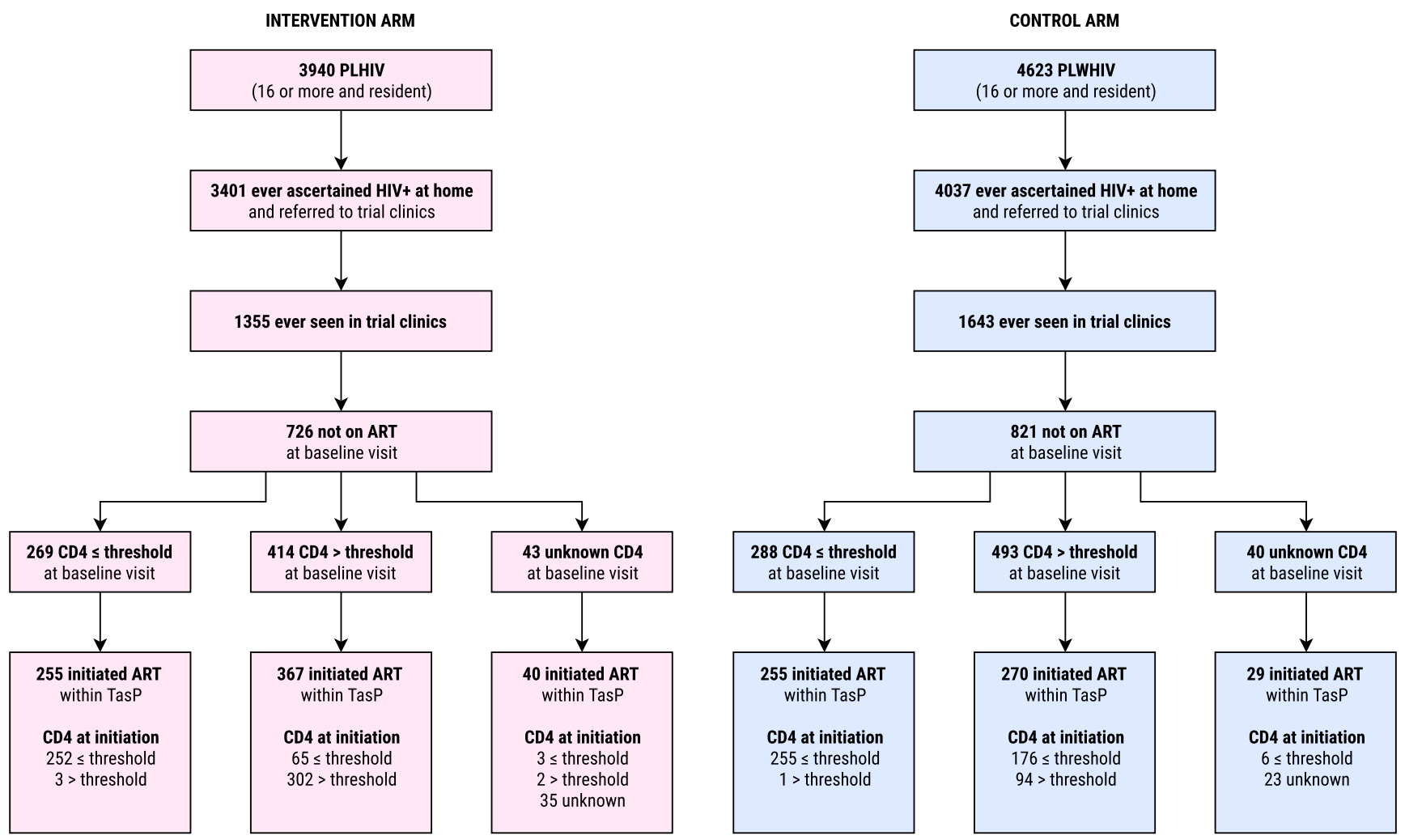

Figure 2. Referral to trial clinics, entry into care, ART status at clinic entry, CD4 count and ART initiation by trial arm, ANRS 12249 TasP trial (2012 to 2016).

Threshold was equal to 350 cells $/ \mathrm{mm}^{3}$ before 1 January 2015, and to 500 cells $/ \mathrm{mm}^{3}$ thereafter. 
not change significantly over the course of the trial (Figure S1)

Among the PLHIV population (Figure 2), 7438 (87\%) have ever been contacted, ascertained HIV positive and referred to trial clinics by a fieldworker; 2998 (35\% of all PLHIV) visited trial clinics at least once; 1547 (18\%) were not on ART at the first clinic visit. In intervention arm, among the 414 patients with a CD4 count above national guidelines threshold at baseline clinic visit, 367 (89\%) initiated ART within trial clinics, including 65 whose CD4 count was below the threshold at the time of ART initiation (due to a decrease in CD4 count or to the change of national guidelines between baseline visit and ART initiation). In the control arm, among the 493 patients with a CD4 count at baseline above the threshold, 270 (55\%) initiated ART at a later point within the trial: 176 patients with a CD4 count below the threshold at initiation and 94 with a CD4 count still above (but with potential other indications for treatment).

At cluster opening, PVS was on average lower in the intervention arm than in the control arm $(-2.5 \%, p=0.180$, Table 1 and Figure 3, detailed HIV care cascade in Figures S2, S3 and S4). Between cluster opening and January 1, 2016, PVS increased significantly in both arms (intervention: $23.5 \%$ to $46.2 \%$, $+22.8, p<0.001$; control: $26.0 \%$ to $44.6 \%$, +18.6 , $p<0.001)$. The increase in the intervention arm was slightly higher than in the control arm but not significantly (difference-in-differences: $+4.2 \%, p=0.258$ ), due to a null effect of trial arm on PVS increase (ANCOVA, $p=0.514$ ), resulting in similar PVS by arm at trial end (difference: 1.6\%, $p=0.635$ ). When stratifying by year of cluster opening, the increase was similar between arms for the four clusters opened in 2012 ( $p=0.475$, Table 1 ) and for the 12 clusters opened in 2014 ( $p=0.745$, Table 1$)$. The PVS increase was significantly higher in the intervention arm only for the six clusters opened in 2013 (+30.5\% vs. $+19.8 \%, p=0.033$, Table 1$)$.

In multivariate model 1 (Table 2), the increase of PVS was mainly associated with years since cluster opening ( $+4.5 \%$ per year [95\% Cl: +3\%; +6\%], $p<0.001)$. There was also some association with calendar time (+1.9\% [0\%; +3\%], $p=0.012$ ). At cluster opening, PVS was lower in intervention arm but not significantly $(-1.3 \%[-6 \% ;+3 \%], p=0.554)$. PVS increased faster but not significantly in intervention arm (interaction term: $+2.4 \%[-1 \% ;+6 \%], p=0.131)$. When controlling for sociodemographic changes and HIV prevalence at the cluster level (model 2), the interaction term became statistically significant ( $p=0.021$ ). The only sociodemographic covariate with a significant effect is the proportion of individuals being 60 years old or more within the cluster $(p=0.030)$. Model 2 suggests that, after controlling for sociodemographic changes and calendar trends, and every year, PVS increased by $+4.4 \%$ in the control arm and by $+7.0 \%(4.4 \%+2.6 \%)$ in the intervention arm.

Disaggregated results by HIV-care continuum steps are in Figure 4. Regarding the proportion of PLHIV diagnosed, the association with years since cluster opening was not significant (Table S1), that proportion being already high (>75\%) at baseline in all clusters (Figure S5). The proportion of being in HIV care among those diagnosed (Figure S6) remained almost stable over time, resulting in a negative association with calendar years counterbalanced by a positive association with years since cluster opening (Table S2). The increase was also significantly faster in the intervention arm. The proportion being on ART among those being in HIV care increased over time (Figure S7), the positive association of calendar years being higher than the positive association of years since cluster opening (Table S3). For that step, baseline values were higher in the intervention arm, resulting in a slower increase with years since cluster opening compared to the control arm. Finally, the proportion virally suppressed among those on ART was also high at baseline (Figure S8). There was no significant association with calendar time (Table S4) but a significant association with years since cluster opening after the first year of trial implementation.

Results remained consistent when disaggregating the effect of calendar years and years since cluster opening by period to consider potential non-linear effects (Table S5 and Figure S9).

In our sensitivity analyses, accounting for individuals with no observed HIV status (Figure S5), PVS was re-estimated (Figures S9 and S10). In approach A, where those imputed to be HIV positive were considered as not being virally suppressed; model results remained unchanged (Figure S11 and Figure S12). In approach B where cascade status was also imputed by modelling, coefficients remained similar except that the association with calendar years disappeared, the association with time since cluster opening decreased slightly and the additional association with time since cluster opening in trial arm increased (Figure S11 and Figure S11).

\section{DISCUSSION}

PVS increased significantly during the trial (+19\% in the control arm and $+23 \%$ in the intervention arm) and that increase was mainly driven by years since cluster opening, measuring the impact of repeat home-based HIV testing and implementation of local trial clinics, both having been implemented in all clusters. As the majority (>80\%) of PLHIV was already diagnosed at the trial beginning, the association with RHBCT was stronger on linkage-to-care. Previously we showed that RHBCT facilitated re-referral to care of individuals previously in care but lost-to-follow-up. RHBCT was significantly less effective at linking those newly diagnosed [23]. There were also some associations due to contextual changes, measured by calendar years. In particular, in 2015, South Africa changed its treatment initiation guidelines, from a CD4 count of 350 to 500 cells $/ \mathrm{mm}^{3}$, affecting pre-ART patients followed in the control arm and/or local governmental clinics.

While the TasP interventions had a positive effect on PVS in both trial arms, no significant difference was observed between arms at cluster opening, at the end of the trial or in terms of temporal trends, although PVS was slightly lower at cluster opening and increased slightly faster in the intervention arm. A faster increase in the intervention arm was statistically significant only for the third/fourth year of implementation, once controlling for sociodemographic changes at the cluster level.

This result could partly explain why the trial did not demonstrate a significant difference in the cumulative HIV incidence between trial arms: $2.11 \%$ in the intervention arm and $2.27 \%$ in the control arm (adjusted hazard ratio: 1.01 [95\% Cl: 0.87 to 1.17], $p=0.89$ ) [11]. Despite a higher proportion of HIV positive individuals becoming aware of their HIV status as a 
Table 1. Population viral suppression by trial arm, at cluster opening and as of 1 January, 2013, 2014, 2015 and 2016, stratified by year of cluster opening, ANRS 12249 TasP trial

\section{Intervention arm percent $(\mathrm{n} / \mathrm{N})$}

Clusters opened in $2012(2 \times 2)$

Dates

Cluster opening

1 January 2013

1 January 2014

1 January 2015

1 January 2016

Difference in proportions [95\% Cl], p-value

1 January 2014 versus 1 January 2013

1 January 2015 versus 1 January 2014

1 January 2016 versus 1 January 2015

1 January 2016 versus 1 January 2013

1 January 2016 versus cluster opening

Clusters opened in $2013(2 \times 3)$

Dates

Cluster opening

1 January 2014

1 January 2015

1 January 2016

Difference in proportions [95\% Cl], p-value

1 January 2015 versus 1 January 2014

1 January 2016 versus 1 January 2015

1 January 2016 versus 1 January 2014

1 January 2016 versus cluster opening

Clusters opened in $2014(2 \times 6)$

Dates

Cluster opening

1 January 2015

1 January 2016

Difference in proportions [95\% Cl], $p$-value

1 January 2016 versus 1 January 2015

1 January 2016 versus cluster opening

All cluster groups combined $(2 \times 11)$

Dates

Cluster opening
1 January 2015
1 January 2016

Difference in proportions [95\% Cl], p-value

1 January 2016 versus 1 January 2015

1 January 2016 versus cluster opening

$$
\begin{aligned}
& 24.7 \%(98 / 396) \\
& 29.0 \%(119 / 410) \\
& 36.0 \%(151 / 420) \\
& 41.6 \%(178 / 428) \\
& 49.1 \%(189 / 385)
\end{aligned}
$$

$+6.9 \%[-23.1 ; 36.9]$
$+5.6 \%[5.1 ; 6.2],<0.0$
$+7.5 \%[-8.4 ; 23.4]$
$+20.1 \%[12.9 ; 27.2]$
$+24.3 \%[18.3 ; 30.3]$

22.9\% (175/763)
$34.6 \%(357 / 1032)$
$43.7 \%(479 / 1095)$
$53.5 \%(530 / 991)$

$+9.2 \%[4.0 ; 14.3],<0.001 * * *$

$+9.7 \%[-4.2 ; 23.7], 0.250$

$+18.9 \%[15.4 ; 22.4],<0.001 * * *$

$+30.5 \%$ [21.2; 39.9], <0.001***

$23.4 \%(355 / 1517)$

$26.5 \%(422 / 1590)$

$40.7 \%(614 / 1507)$

$+14.2 \%$ [11.0; 17.4], 0.033*

$+17.3 \%[14.8 ; 19.9],<0.001 * * *$

$23.5 \%(628 / 2676)$

$34.7 \%(1079 / 3113)$

46.2\% (1333/2883)

$+11.6 \%[7.9 ; 15.3],<0.001 * * *$ $+22.8 \%[16.7 ; 28.9],<0.001 * * *$

\section{Control arm percent $(\mathrm{n} / \mathrm{N})$}

intervention versus control

[95\% Cl], $p$-value
Difference in proportions
$26.0 \%(320 / 1233)$

$30.8 \%(460 / 1493)$

$36.6 \%(584 / 1595)$

$45.8 \%(643 / 1405)$

$+5.8 \%[3.3 ; 8.3],<0.001 * * *$
$+9.2 \%[5.7 ; 12.6],<0.001 * * *$
$+15.0 \%[11.8 ; 18.1],<0.001 * * *$
$+19.8 \%[7.1 ; 32.5],<0.001 * * *$

$26.6 \%(419 / 1576)$

$28.9 \%(478 / 1656)$

$42.7 \%(713 / 1668)$

$+13.9 \%$ [5.9; 21.9], <0.001***

$+16.2 \%[7.5 ; 24.9],<0.001 * * *$

26.0\% (801/3079)

$33.2 \%(1190 / 3585)$

$44.6 \%(1486 / 3331)$
$-3.0 \%[-12.1 ; 6.1], 0.519$

$+3.8 \%[-8.0 ; 15.6], 0.382$

$+7.1 \%[-28.7 ; 43.0], 0.265$

$+7.7 \%[-3.6 ; 19.1], 0.063$

Difference in differences

[95\% Cl], $p$-value

$+3.3 \%[-3.8 ; 10.5], 0.257$

$+0.6 \%[-15.1 ; 16.3], 0.814$

$+3.9 \%[0.0 ; 7.8], 0.033 *$

$+10.7 \%$ [0.6; 20.8], 0.033*

$-3.2 \%$ [-9.6; 3.2], 0.251

$-2.3 \%$ [-8.0; 3.4], 0.377

$-2.0 \%$ [-8.2; 4.2], 0.505

Difference in differences

[95\% Cl], p-value

$+0.3 \%[-7.5 ; 8.1], 0.938$

$+1.2 \%[-7.3 ; 9.7], 0.745$

$-2.5 \%[-6.5 ; 1.4], 0.180$

$+1.5 \%[-7.4 ; 10.4], 0.739$

$+1.6 \%[-5.4 ; 8.6], 0.651$

Difference in differences

[95\% Cl], $p$-value

$+0.2 \%[-4.4 ; 4.7], 0.947$

$+4.2 \%[-2.8 ; 11.1], 0.258$
$+11.4 \%[7.5 ; 15.3],<0.001 * * *$ $+18.6 \%[14.4 ; 22.8],<0.001 * * *$

Cluster opening is different for each cluster. p-value: $* * *<0.001<* *<0.01<*<0.05$. 
(a) by calendar time

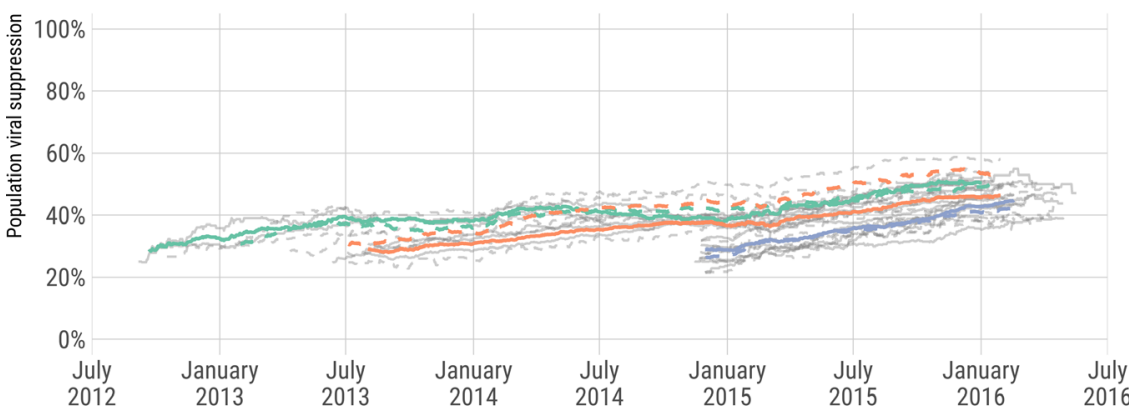

(b) by time since cluster opening

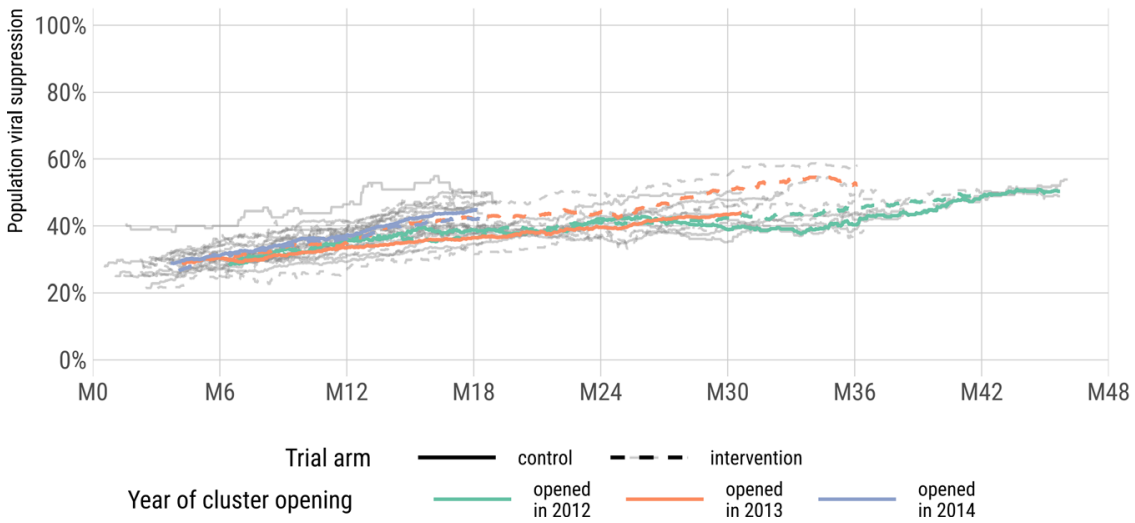

Figure 3. Population viral suppression over calendar time (a) and time since cluster opening (b), by cluster, year of cluster opening and trial arm, ANRS 12249 TasP trial (2012 to 2016).

Each grey line represents a different cluster.

Table 2. Temporal trends of population viral suppression (multivariate analysis), ANRS 12249 TasP trial (2012 to 2016)

\begin{tabular}{|c|c|c|c|c|}
\hline \multirow[b]{2}{*}{ Variable } & \multicolumn{2}{|l|}{ Model 1} & \multicolumn{2}{|l|}{ Model 2} \\
\hline & Estimate $[95 \% \mathrm{Cl}]$ & $p$-value & Estimate $[95 \% \mathrm{Cl}]$ & $p$-value \\
\hline Time since cluster opening (annual increase) ${ }^{a}$ & $0.045[0.03 ; 0.06]$ & $<0.001$ & $0.044[0.02 ; 0.07]$ & $<0.001$ \\
\hline Intervention arm (vs. control, at cluster opening) & $0.013[-0.06 ; 0.03]$ & 0.554 & $-5.031[-0.07 ; 0.01]$ & 0.090 \\
\hline Proportion of 16 to 29 years old (within cluster) ${ }^{b}$ & & & $-0.036[-0.46 ; 0.39]$ & 0.868 \\
\hline Proportion of 60 or more years old (within cluster) ${ }^{b}$ & & & $1.332[0.11 ; 2.56]$ & 0.030 \\
\hline Proportion with at least secondary level of education (within cluster) ${ }^{b}$ & & & $-0.013[-0.32 ; 0.30]$ & 0.930 \\
\hline Proportion being employed (within cluster) ${ }^{\mathrm{b}}$ & & & $0.726[-0.05 ; 1.50]$ & 0.065 \\
\hline Proportion being student (within cluster) ${ }^{\mathrm{b}}$ & & & $-0.171[-0.67 ; 0.33]$ & 0.499 \\
\hline
\end{tabular}

Model 1 is adjusted on calendar time, time since cluster opening and trial arm. Model 2 is also adjusted on cluster-level sociodemographic characteristics. Models are computed at cluster-day level.

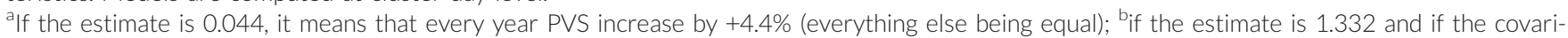
ate increases by 0.1 (i.e. by $10 \%$, for example from $20 \%$ to $30 \%$ ), everything else being equal, PVS would increase by $0.1 \times 1.332=0.1332$, that is, by $13.3 \%$

result of the trial, a high proportion of those initiating ART achieving virological suppression [11], high levels of treatment adherence [24] and good retention into care [25], low linkage to care was observed [13,23]. As a result, ART initiation remained similar between arms: all ART initiations within trial clinics represent $17 \%(662 / 3940)$ of the PLHIV population in 
the intervention arm, compared with 12\% (554/4623) in the control arm. In addition, the PLHIV population turnover in the trial area was high, more than one fifth being replaced every year, mainly due to out- and in-migration as well as the continuous inflow of new HIV infections, attenuating the impact of the interventions on the cascade of HIV care and subsequently the differences between arms [26].

Tanser et al., using data from another rural community in KwaZulu-Natal, found that HIV incidence was not directly associated with PVS but rather with population viral load metrics that account for HIV prevalence [27]. As HIV prevalence remained almost stable during the trial, we cannot exclude the possibility that HIV incidence may have been decreasing in both arms. Unfortunately, the trial was not powered to estimate temporal trends of HIV incidence.

Although the data collected through the ANRS 12249 TasP trial allowed us to analyse the HIV care cascade at a fine level, there are some limitations. The position within the continuum of HIV care is known very precisely for those followed in trial clinics, meanwhile proxy indicators were used to estimate care status and ART status for those followed in local governmental clinics. Our estimation of entry into care (based on CD4 counts and viral loads) is robust. However, the identification of when care was exited is less precise for patients matched to the NHLS database. Limitations of the matching algorithm have been discussed in detail elsewhere [26]. In addition, we had no data for those receiving HIV care in clinics outside the Hlabisa sub-district or in the private sector. Our estimated proportions of PLHIV being in care and virally suppressed should thus be considered as lower bounds.

The three other UTT cluster-randomized trials conducted in eastern and southern Africa. have reported their main findings. In the BCPP trial in Botswana (2013 to 2018), cumulative HIV incidence was significantly reduced by $30 \%$ in the intervention arm [28]. PVS increased from 75\% to 82\% (+7) in the control arm and from $70 \%$ to $88 \%(+18)$ in the intervention $\operatorname{arm}(p<0.001)$. In the SEARCH trial in Kenya and Uganda (2013 to 2017), PVS increased from $42 \%$ to $68 \%$ $(+26)$ in the control arm and from $42 \%$ to $79 \%(+37)$ in the intervention arm (risk ratio: 1.11, $p<0.001$ ) [29]. Cumulative HIV incidence was not significantly different between arms, but a 32\% decline was observed between the first and third year. Rapid improvement of the cascade within the control arm, new treatment guidelines and high level of mobility were mentioned explaining the null effect between arms. Finally, the main results from the HPTN 071 PopART trial conducted in Zambia and South Africa (2013 to 2018) showed a reduction in HIV incidence of $7 \%$ (not significant) and 30\% ( $p=0.006)$

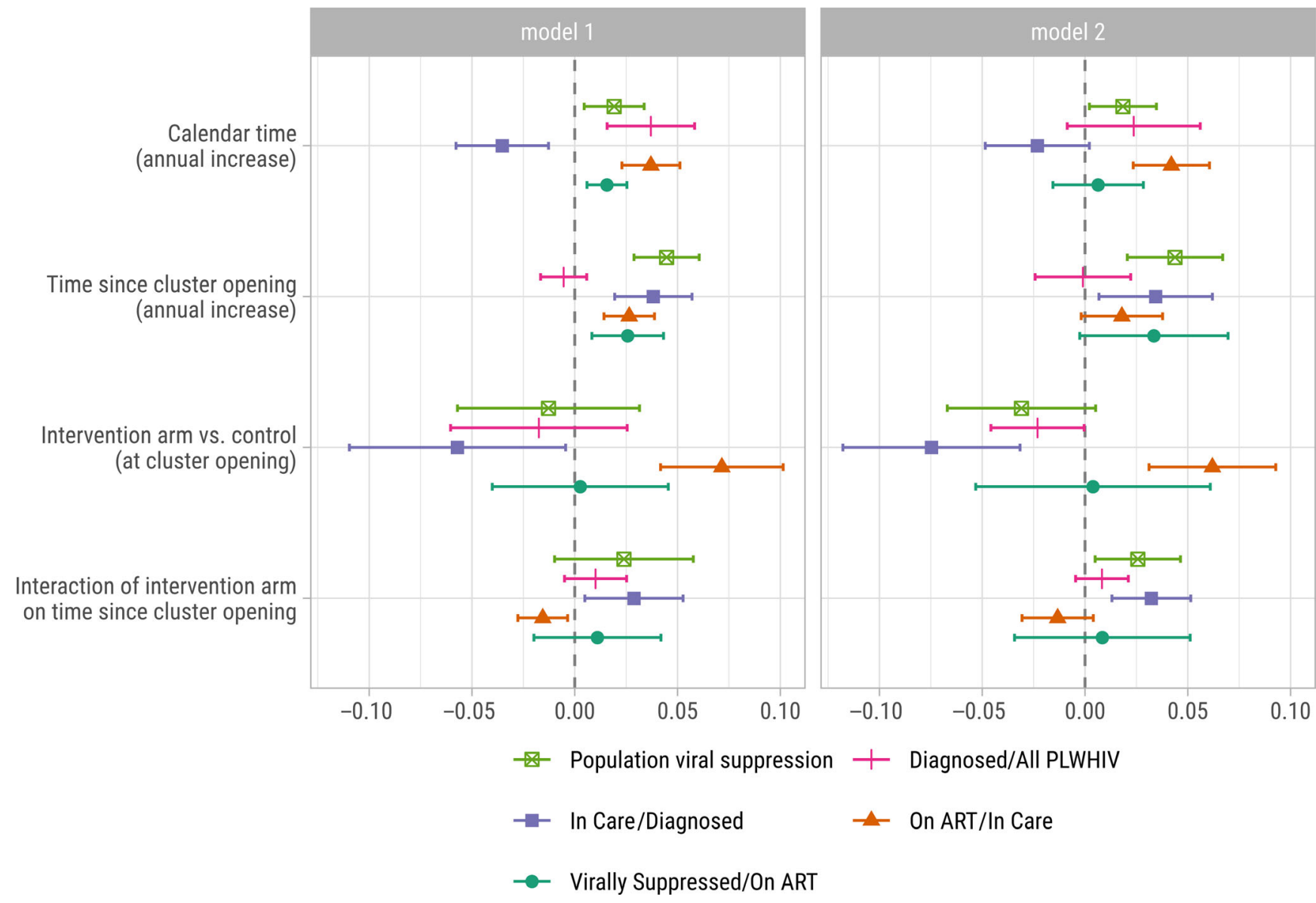

Figure 4. Effect of calendar time, time since cluster opening and trial arm on the different subcomponents of the HIV care cascade, ANRS 12249 TasP trial (2012 to 2016).

Model 1 is adjusted on calendar time, time since cluster opening and trial arm. Model 2 is also adjusted on cluster-level sociodemographic characteristics. 
in their two intervention arms compared to their control arm [30]. After two years of intervention, PVS increased from 56\% to $72 \%(+16$, adjusted prevalence ratio: $1.16, p=0.07)$ and from $57 \%$ to $68 \%(+11$, aPR: $1.08, p=0.30)$ in their intervention arms versus $54 \%$ to $60 \%(+6 \%)$ in the control arm. Specific interventions aiming to improve HIV testing and linkage-tocare were implemented in both arms for TasP and SEARCH trials while they were implemented only in the intervention arms for BCPP and PoPART [10], but precise interventions varied according to the trial and could explain the difference of PVS increase between the trials. A reduction in cumulative HIV incidence was observed only for trials where testing and linkage was not enhanced in the control arm.

\section{CONCLUSION}

Viral suppression at the population level was improved significantly but similarly in both trial arms. The null effect in terms of cumulative incidence in TasP trial between arms does not mean that universal ART does not reduce the risk of population-level HIV acquisition, but rather that gains due to changes in ART-initiation guidelines alone are relatively small compared to gains obtained by strategies to maximize testing and linkage to care, HIV testing and linkage to care having been also scaled up in the control arm. The achievement of the 90-90-90 targets will not be met if the operational and implementational challenges limiting access to care and treatment, often context-specific, are not properly addressed.

\section{AUTHORS' AFFILIATIONS}

${ }^{1}$ Centre Population et Développement, Institut de Recherche pour le Développement, Université Paris Descartes, Inserm, Paris, France; ${ }^{2}$ Africa Health Research Institute, KwaZulu-Natal, South Africa; ${ }^{3}$ Africa Health Research Institute, School of Nursing and Public Health, University of KwaZulu-Natal, KwaZulu-Natal, South Africa; ${ }^{4}$ Faculty of Medicine and Faculty of Social Sciences, University of Southampton, Southampton, United Kingdom; ${ }^{5}$ Research Department of Infection and Population Health, University College London, London, United Kingdom; ${ }^{6}$ Department of Global Health \& Infection, Brighton and Sussex Medical School, Brighton, United Kingdom; ${ }^{7}$ School of Public Health (ISPED), Inserm, Bordeaux Population Health Research Center, UMR 1219, Bordeaux University, Bordeaux, France; ${ }^{8}$ Department of Global Health \& Population, Harvard School of Public Health, Harvard University, Boston, USA; ${ }^{9}$ Faculty of Medicine, Institute of Public Health, Heidelberg University, Heidelberg, Germany; ${ }^{10}$ Division of Infection and Immunity, University College London, London, United Kingdom

\section{COMPETING INTEREST}

$\mathrm{Cl}$ has received honoraria for services rendered to Gilead Sciences. All other authors declare no competing interests.

\section{AUTHORS' CONTRIBUTIONS}

Cl, JOG, DP and FD designed and implemented the ANRS 12249 TasP trial. JL, JOG and NM developed the research question addressed in this paper. $J \mathrm{~L}$ and MHD did the statistical analysis. $J L$ wrote the first draft of the manuscript. All authors contributed to the interpretation and presentation of the findings. All authors approved the final version of the manuscript for submission. The content is solely the responsibility of the authors and does not represent the official views of 3ie or the Bill \& Melinda Gates Foundation.

\section{ACKNOWLEDGEMENTS}

We thank the study volunteers for allowing us into their homes and participating in this trial, the KwaZulu-Natal Provincial and the National Department of Health of South Africa for their support of this study. We thank the staff of the Africa Health Research Institute for the trial implementation and analysis of data, including those who did the fieldwork, provided clinical care, developed and maintained the database, entered the data, and verified data quality.

\section{FUNDING}

This trial was sponsored by the French National Agency for AIDS and Viral Hepatitis Research (ANRS; grant number, 2011-375), and funded by the ANRS, the Deutsche Gesellschaft für Internationale Zusammenarbeit (GIZ; grant number, 81151938), and the Bill \& Melinda Gates Foundation through the 3ie Initiative. This trial was done with the support of Merck and Gilead Sciences, which provided the Atripla drug supply. The Africa Health Research Institute, (previously Africa Centre for Population Health, University of KwaZulu-Natal, South Africa) receives core funding from the Wellcome Trust, which provides the platform for the population-based and clinic-based research at AHRI.

\section{THE MEMBERS OF THE ANRS 12249 TASP TRIAL GROUP ARE AS FOLLOWS}

South Africa Till Barnighausen, Kobus Herbst, Collins Iwuji, Thembisa Makowa, Kevi Naidu, Nonhlanhla Okesola, Tulio de Oliveira, Deenan Pillay, Tamsen Rochat, Frank Tanser, Johannes Viljoen, Thembelihle Zuma (Africa Health Research Institute (previously Africa Centre for Population Health, University of KwaZulu-Natal), KwaZulu-Natal, Durban). Frank Tanser, Nuala McGrath (School of Nursing and Public Health, University of KwaZulu-Natal, KwaZulu-Natal, Durban). Tulio de Oliveira (Nelson R Mandela School of Medicine, College of Health Sciences, University of KwaZulu-Natal, KwaZulu-Natal, Durban). France Eric Balestre, Francois Dabis, Sophie Karcher, Joanna Orne-Gliemann, Melanie Plazy, Melanie Prague, Rodolphe Thiebaut, Thierry Tiendrebeogo (ISPED, Centre INSERM U1219 Bordeaux Population Health, Universite de Bordeaux, Bordeaux). Sylvie Boyer, Hermann Donfouet, Andrea Gosset, Laura March, Camelia Protopopescu, Bruno Spire (INSERM, UMR912 SESSTIM, Universite Aix Marseille, Marseille). Joseph Larmarange, Maxime Inghels, Hassimiou Diallo (Centre Population et Développement UMR 196, Universite Paris Descartes, Institut de Recherche pour le Développement, Paris). Vincent Calvez, Anne Derache, AnneGenevieve Marcelin (AP-HP, Virology, Hopital Pitie-Salpetriere, INSERM-Sorbonne Universites, UPMC Univ Paris 06, UMR-S 1136, Paris). Rosemary DraySpira, France Lert, Kamal El Farouki (INSERM U1018, CESP, Epidemiology of Occupational and Social Determinants of Health, Villejuif). Marie-Laure Chaix (EA 3620, Universite Paris-Descartes, Laboratoire de Virologie, Hopital NeckerEnfants Malades, AP-HP, Paris). Brigitte Bazin, Claire Rekacewicz (sponsor representatives; ANRS, Paris). UK Collins Iwuji, John Imrie (Department of Infection and Population Health, University College London, London). Deenan Pillay (Division of Infection and Immunity, University College London, London). Nuala McGrath (Department of Epidemiology and Public Health, University College London, London). Richard Lessells (Department of Clinical Research, London School of Hygiene \& Tropical Medicine, London). Collins Iwuji (Department of Global Health and Infection, Brighton and Sussex Medical School, University of Sussex, Brighton). Nuala McGrath (Academic Unit of Primary Care and Population Sciences, and Department of Social Statistics and Demography, University of Southampton, Southampton). Colin Newell (Academic Unit of Human Development and Health, University of Southampton, Southampton). Marie-Louise Newell, (Academic Unit of Human Development and Health, and Global Health Research Institute, University of Southampton, Southampton). Switzerland Alexandra Calmy (Service des Maladies Infectieuses, HIV Unit, Hopitaux Universitaires de Geneve, Geneva). USA Kenneth Freedberg (Massachusetts General Hospital, Harvard Medical School, Harvard University, Boston, MA). Till Barnighausen (Department of Global Health and Population, Harvard School of Public Health, Harvard University, Boston, MA). Netherlands Jan Hontelez (Department of Public Health, Erasmus MC, Erasmus University Medical Center Rotterdam, Rotterdam). Germany Till Barnighausen, Jan Hontelez (Institute of Public Health, Faculty of Medicine, Heidelberg University, Heidelberg).

\section{REFERENCES}

1. Danel C, Moh R, Gabillard D, Badje A, Le Carrou J, Ouassa T, et al. A trial of early antiretrovirals and isoniazid preventive therapy in Africa. N Engl J Med. 2015;373(9):808-22.

2. INSIGHT START Study Group, Lundgren JD, Babiker AG, Gordin F, Emery S, Grund $B$ et al. Initiation of antiretroviral therapy in early asymptomatic HIV infection. N Engl J Med. 2015;373(9):795-807. 
3. Cohen MS, Chen YQ, McCauley M, Gamble T, Hosseinipour MC, Kumarasamy $\mathrm{N}$, et al. Prevention of HIV-1 infection with early antiretroviral therapy. N Engl J Med. 2011;365(6):493-505.

4. Granich RM, Gilks CF, Dye C, De Cock KM, Williams BG. Universal voluntary HIV testing with immediate antiretroviral therapy as a strategy for elimination of HIV transmission: a mathematical model. Lancet. 2009;373:48-57.

5. Tanser F, Barnighausen T, Grapsa E, Zaidi J, Newell M-L. High coverage of ART associated with decline in risk of HIV acquisition in rural KwaZulu-Natal, South Africa. Science. 2013;339(6122):966-71.

6. Gardner EM, McLees MP, Steiner JF, del Rio C, Burman WJ. The spectrum of engagement in HIV care and its relevance to test-and-treat strategies for prevention of HIV infection. Clin Infect Dis. 2011;52(6):793-800.

7. Miller WC, Lesko CR, Powers KA. The HIV care cascade: simple concept, complex realization. Sex Transm Dis. 2014;41(1):41-2.

8. Iwuji CC, Orne-Gliemann J, Tanser F, Boyer S, Lessells RJ, Lert F, et al. Evaluation of the impact of immediate versus $\mathrm{WHO}$ recommendations-guided antiretroviral therapy initiation on HIV incidence: the ANRS 12249 TasP (Treatment as Prevention) trial in Hlabisa sub-district, KwaZulu-Natal, South Africa: study protocol for a cluster randomised controlled trial. Trials. 2013;14(1):230.

9. Hayes R, Ayles H, Beyers N, Sabapathy K, Floyd S, Shanaube K, et al. HPTN 071 (PopART): rationale and design of a cluster-randomised trial of the population impact of an HIV combination prevention intervention including universal testing and treatment - a study protocol for a cluster randomised trial. Trials. 2014;15(1):57.

10. Delphine Perriat, Laura Balzer, Richard Hayes, Shahin Lockman, Fiona Walsh, Helen Ayles, et al. Comparative assessment of five trials of universal HIV testing and treatment in sub-Saharan Africa. J Int AIDS Soc. 2018;21:e25048. 11. Iwuji CC, Orne-Gliemann J, Larmarange J, Balestre E, Thiebaut R, Tanser F, et al. Universal test and treat and the HIV epidemic in rural South Africa: a phase 4, open-label, community cluster randomised trial. Lancet HIV. 2018;5(3): e116-25

12. Zaidi J, Grapsa E, Tanser F, Newell M-L, Bärnighausen T. Dramatic increase in HIV prevalence after scale-up of antiretroviral treatment. AIDS. 2013;27 (14):2301-5.

13. Iwuji CC, Orne-Gliemann J, Larmarange J, Okesola N, Tanser F, Thiebaut R, et al. Uptake of home-based HIV testing, linkage to care, and community attitudes about ART in Rural KwaZulu-Natal, South Africa: descriptive results from the first phase of the ANRS 12249 TasP Cluster-Randomised Trial. PLoS Med. 2016;13:e1002107.

14. Muhwava W, Hosegood V, Nyirenda M, Newell C, Herbst K, Newell M-L. Levels and determinants of population migration in rural KwaZulu-Natal, South Africa. Afr Popul Stud. 2010;24(3):259-80.

15. Camlin CS, Hosegood V, Newell M-L, McGrath N, Bärnighausen T, Snow RC. Gender, migration and HIV in rural KwaZulu-Natal, South Africa. PLoS ONE. 2010;5:e11539.

16. Hosegood V, McGrath N, Moultrie TA. Dispensing with marriage: Marital and partnership trends in rural KwaZulu-Natal, South Africa 2000-2006. Demogr Res. 2009;20(20):279-312.

17. Orne-Gliemann J, Larmarange J, Boyer S, Iwuji C, McGrath N, Bärnighausen T, et al. Addressing social issues in a universal HIV test and treat intervention trial (ANRS 12249 TasP) in South Africa: methods for appraisal. BMC Public Health. 2015;15(1):209.

18. Houlihan CF, Bland RM, Mutevedzi PC, Lessells RJ, Ndirangu J, Thulare H, et al. Cohort profile: hlabisa HIV treatment and care programme. Int J Epidemiol. 2011;40(2):318-26.

19. UNAIDS. 90-90-90: An ambitious treatment target to help end the AIDS epidemic [Internet]. Geneva: UNAIDS; 2014 [cited 2017 Oct 11]. Report No.: UNAIDS/JC2684. Available from: http://www.unaids.org/sites/default/files/med ia_asset/90-90-90_en_0.pdf

20. Frison L, Pocock SJ. Repeated measures in clinical trials: Analysis using mean summary statistics and its implications for design. Stat Med. 1992;11 (13):1685-704.

21. R Core Team. A language and environment for statistical computing [Internet]. Vienna, Austria: R Foundation for Statistical Computing; 2018 [cited 2018 Jun 29]. Available from: http://www.R-project.org

22. Cameron AC, Gelbach JB, Miller DL. Bootstrap-based improvements for inference with clustered errors. Rev Econ Stat. 2008;90(3):414-27.

23. Plazy M, Farouki KE, Iwuji C, Okesola N, Orne-Gliemann J, Larmarange J, et al. Access to HIV care in the context of universal test and treat: challenges within the ANRS 12249 TasP cluster-randomized trial in rural South Africa. J Int AIDS Soc [Internet]. 2016 [cited 2016 Jun 1];19(1). Available from: http:// www.jiasociety.org/index.php/jias/article/view/20913

24. Iwuji C, McGrath N, Calmy A, Dabis F, Pillay D, Newell M-L, et al. Universal test and treat is not associated with sub-optimal antiretroviral therapy adherence in rural South Africa: the ANRS 12249 TasP trial. J Int AIDS Soc. 2018;21:e25112.

25. Gosset A, Protopopescu C, Larmarange J, Orne-Gliemann J, McGrath N, Pillay D, et al. Retention in care trajectories of HIV-positive individuals participating in a universal test and treat programme in rural South Africa (ANRS 12249 TasP trial). J Acquir Immune Defic Syndr. 2019;80(4):375-85.

26. Larmarange J, Diallo MH, McGrath N, Iwuji C, Plazy M, Thiébaut R, et al. The impact of population dynamics on the population HIV care cascade: results from the ANRS 12249 Treatment as Prevention trial in rural KwaZulu-Natal (South Africa). J Int AIDS Soc. 2018;21:e25128.

27. Tanser F, Vandormael A, Cuadros D, Phillips AN, de Oliveira T, Tomita A, et al. Effect of population viral load on prospective HIV incidence in a hyperendemic rural African community. Sci Transl Med. 2017;9(420):eaam8012.

28. Makhema J, Wirth KE, Pretorius Holme M, Gaolathe T, Kadima E, Chakalisa $U$, et al.Impact of prevention and treatment interventions on population HIV incidence: Primary results of Botswana Combination Prevention Project (BCPP / Ya Tsie) (\#WEAX0105LB). In Amsterdam; 2018 [cited 2018 Sep 24]. Available from: http://programme.aids2018.org/Abstract/Abstract/13216

29. Havlir D, Charlebois E, Balzer L, Clark TD, Kwarisiima D, Ayieko J, et al.SEARCH community cluster randomized study of HIV "test and treat" using multi- disease approach and streamlined care in rural Uganda and Kenya. In Amsterdam; 2018 [cited 2018 Sep 24]. Available from: http://programme.a ids2018.org/Abstract/Abstract/13469

30. Hayes RJ, Donnell D, Floyd S, Mandla N, Bwalya J, Shanaube K, et al. Impact of universal testing and treatment in Zambia and South Africa: HPTN071 (PopART). In Seattle; 2019.

\section{SUPPORTING INFORMATION}

Additional information may be found under the Supporting Information tab for this article.

Figure S1. Observed HIV prevalence among all resident adult population over calendar time and time since cluster opening, by cluster, year of cluster opening and trial arm, ANRS 12249 TasP trial (2012 to 2016). Each grey line represents a different cluster.

Figure S2. HIV care cascade by trial arm, pre-intervention and as of 1 January 2013, 2014, 2015 and 2016, stratified by year of cluster opening, ANRS 12249 TasP trial.

Figure S3. HIV care cascade by trial arm according to calendar time, stratified by year of cluster opening, ANRS 12249 TasP trial (2012 to 2016). The figure starts at the end of the initial population census (first survey round) and stops at the beginning of the last survey round.

Figure S4. HIV care cascade by trial arm according to time since cluster opening, stratified by year of cluster opening, ANRS 12249 TasP trial (2012 to 2016). The figure starts at the end of the initial population census (first survey round) and stops at the beginning of the last survey round.

Figure S5. Proportion being diagnosed among all resident PLHIV over calendar time and time since cluster opening, by cluster, year of cluster opening and trial arm, ANRS 12249 TasP trial (2012 to 2016). Each grey line represents a different cluster.

Figure S6. Proportion being in care among those being diagnosed over calendar time and time since cluster opening, by cluster, year of cluster opening and trial arm, ANRS 12249 TasP trial (2012 to 2016). Each grey line represents a different cluster.

Figure S7. Proportion being on ART among those in care over calendar time and time since cluster opening, by cluster, year of cluster opening and trial arm, ANRS 12249 TasP trial (2012 to 2016). Each grey line represents a different cluster. 
Figure S8. Proportion being virally suppressed among those on ART over calendar time and time since cluster opening, by cluster, year of cluster opening and trial arm, ANRS 12249 TasP trial (2012 to 2016). Each grey line represents a different cluster.

Figure S9. Proportion of individuals with an unknown HIV status among all resident adult population over calendar time and time since cluster opening, by cluster, year of cluster opening and trial arm, ANRS 12249 TasP trial (2012 to 2016). Each grey line represents a different cluster.

Figure S10. Imputed population viral suppression (approach A) over calendar time and time since cluster opening, by cluster, year of cluster opening and trial arm, ANRS 12249 TasP trial (2012 to 2016). Each grey line represents a different cluster. HIV status was imputed for those with no observed data. Those predicted to be HIV positive were considered as not virally suppressed.

Figure S11. Imputed population viral suppression (approach B) over calendar time and time since cluster opening, by cluster, year of cluster opening and trial arm, ANRS 12249 TasP trial (2012 to 2016). Each grey line represents a different cluster. HIV status was imputed for those with no observed data. Cascade status was also imputed for those predicted to be HIV positive.

Figure S12. Comparison of the effect of calendar time, time since cluster opening and trial arm according to three scenarios (sensitivity analysis), ANRS 12249 TasP trial (2012 to 2016). Model 1 is adjusted on calendar time, time since cluster opening and trial arm. Model 2 is also adjusted on clusterlevel sociodemographic characteristics. HIV status was imputed for those with no observed data. Approach A: those predicted to be HIV positive were considered as not virally suppressed. Approach B: cascade status was also imputed for those predicted to be HIV positive.

Figure S13. Effect of calendar time, time since cluster opening and trial arm on the different subcomponents of the HIV care cascade, with three coefficients for calendar time and three coefficients for time since cluster opening, ANRS 12249 TasP trial (2012 to 2016)

Table S1. Temporal trends of the proportion being diagnosed among all resident PLHIV (multivariate analysis), ANRS 12249 TasP trial (2012 to 2016)

Table S2. Temporal trends of the proportion being in care among those being diagnosed (multivariate analysis), ANRS 12249 TasP trial (2012 to 2016)

Table S3. Temporal trends of the proportion being on ART among those in care (multivariate analysis), ANRS 12249 TasP trial (2012 to 2016)

Table S4. Temporal trends of the proportion being virally suppressed among those on ART (multivariate analysis), ANRS 12249 TasP trial (2012 to 2016)

Table S5. Temporal trends of population viral suppression (multivariate analysis) with three coefficients for calendar time and three coefficients for time since cluster opening, ANRS 12249 TasP trial (2012 to 2016)

Table S6. Temporal trends of population viral suppression, according to three scenarios (sensitivity analysis), ANRS 12249 TasP trial (2012 to 2016). Models adjusted on calendar time, time since cluster opening and trial arm and clusterlevel sociod emographic characteristics (model 2). HIV status was imputed for those with no observed data. Approach A: those predicted to be HIV positive were considered as not virally suppressed. Approach B: cascade status was also imputed for those predicted to be HIV positive. 